\section{Everything in the garden...}

Peter D. Moore

The European Garden Flora. Vol.II

Monocotyledons (Part II).

Edited by S.M. Walters et al.

Cambridge University Press: 1984.

Pp.318. £30, \$59.50.

IT MAY seem unlikely, but gardens can be frustrating places for the botanist. The problem is one of taxonomy. Out in the field, things are much easier; whether on the Downs of Sussex or in the swamps of the Nile Delta, there is sure to be a local Flora which permits the identification of just about any plant that you come across. But in the garden the troubles really start, for that tantalizing Saxifraga could have come from almost anywhere, from the Himalayas to Alaska, and where is the Flora which can hope to cover that span? The answer, it seems, lies in The European Garden Flora.

Sponsored by the Royal Horticultural Society and steered by an editorial committee of eminent botanists and horticulturalists, the series seeks to cover all the plants likely to be found in cultivation in Europe, whether in the open or under glass. The only plants deliberately omitted are the crop species and, of course, the weeds.

Such an extraordinarily ambitious project is made no easier by the confused state of horticultural taxonomy and nomenclature: indeed, if the series serves only to untangle some of the classificatory knots and proliferating synonyms it will have achieved a great deal. Add to these problems morphological variations which have resulted from generations of selective breeding in some taxa, and one cannot but admire the vision and determination of those who planned the work.

Volume II (of a total of six) completes the coverage of the Monocotyledons (begun in Vol.I, to be published later this year), and over half of it is devoted to the orchids. For each family a dichotomous key to genera is provided, and a key to families is given at the front of the volume. All keys are arranged in a concise yet clear form, with alternatives placed in a consecutive manner. Technical terms are kept to a minimum and a glossary is provided, as the book is intended to be used by gardeners as well as by taxonomists. This aim is evident also from the text, for the description of each species includes details of where a good illustration can be found, what variants are available in cultivation, and how the species is best grown in Europe. But Europe is a big place and contains a variety of climatic regions which obviously influence what plants can be grown. To overcome the problem, a hardiness code has been devised, based on the mean
January isotherms, and a map of Europe is provided showing their positions. This feature should prove a valuable aid in the selection of species for planting.

A book such as this is not, perhaps, the place to seek personal preferences and opinions from the authors. There are, however, some telling comments, such as " "the unsightly variegated form of Phalaris arundinacea is a depressing feature of many an ill-tended border" - a refreshing reminder that both taxonomists and gardeners are human.

There is a wealth of information here, both scientific and practical. The authors have achieved what might have seemed impossible; they have taken a step towards the relief of the botanist's frustration in the garden and at the same time they have provided the horticulturalist with a concise compendium of modern botanical knowledge.

Peter D. Moore is Senior Lecturer in the Department of Plant Sciences, King's College, University of London.

\section{Pictures in mind}

\section{Stuart Sutherland}

Ghosts in the Mind's Machine: Creating and Using Images in the Brain.

By Stephen Michael Kosslyn.

W.W. Norton: 1984. Pp.249. \$19.95, f15.95.

WHEN asked whether a horse's tail ends above its knee, most people generate a visual image in thinking of the answer. Scientists, artists, engineers and writers constantly use images in their work. Hopes, fears and fantasies are often expressed in images and we cannot escape them even in sleep. Yet until recently there has been little systematic investigation of imagery.

Professor S.M. Kosslyn has put this right by conducting a series of fascinating experiments and proposing a most ingenious theory. The experiments could have been run a hundred years ago, since they do not require any apparatus more elaborate than a few drawings and a timing device. The theory, however, could not have been developed until recently since working out its details required computer simulation. The computer model certainly helped Kosslyn to ask the right questions: the work of his predecessors in the field, such as Francis Galton, is pallid by comparison, possibly because they had no way of formulating a precise theory of imagery that would direct their research. Kosslyn's agreeable book, Ghosts in the Mind's Machine, makes his work accessible to the general reader: it is clearly and simply written, and dispenses with jargon.

One of Kosslyn's more remarkable results is that mental imagery obeys many of the same laws as perception, even when these laws are unknown to the subject. In normally sighted people, acuity for oblique striations is worse than for vertical or horizontal ones. Kosslyn asked subjects to image stripes of a given width at different orientations and to imagine themselves walking backwards away from the stripes: they had to give the distance at which the stripes became so blurred that they could no longer be distinguished. This distance was the same for subjects imaging the stripes as for those actually seeing them and for both groups the oblique stripes blurred at a shorter distance than the horizontal or vertical. Although Kosslyn does not say so, this finding and others he has obtained suggest that the neurophysiological medium responsible for imagery is the same as that used in vision. The low acuity for oblique stripes is thought to be caused by central mechanisms: it would be interesting to discover whether visual phenomena that were purely due to retinal interactions failed to occur in imagery.

Among Kosslyn's other findings are the following. The extent of a visual image is about the same as that of the visual field, and the amount of detail that can be represented declines rapidly from the centre to the outer parts of the image. Subjects can "scan" across an image and the further they have to scan the longer it takes. They can also perform a number of other transformations on the image or on a part of it, including zooming, panning and rotating. He produces evidence to show that an image is built up piece-meal out of separate components - in imaging a person, a broad outline is first constructed and then the details of the face, arms, legs and so on are added.

In his theory, Kosslyn posits that in longterm memory pictorial data is held separately from other forms of knowledge, though the two can interact. In imaging, some of the pictorial data is reproduced in a medium of limited extent which is fine grained in the centre and coarse grained in its outer parts. A procedure called PICTURE recovers the required pictorial data from long-term memory and places it in this medium, starting with a skeleton of the required image and filling in the details of its parts by the use of subsidiary procedures. One of these, named FIND, locates the correct point in the image at which to insert a more detailed representation of its parts: FIND operates by a random search, which seems a little implausible. The same procedure can be used to find a part of an image when information about it is needed, as for example to respond to the question "Do frogs have a tail?". Further procedures, such as SCAN, PAN and ROTATE, apply transforms to the image.

The results of applying the various operations are shown on a VDU, but although this is a good way of displaying them, Kosslyn is careful to point out that the processes underlying images do not have to be laid out in a pictorial space in the 